\title{
ENFOQUES Y FUNDAMENTOS PARA UN MODELO DE REHABILITACIÓN AMBULATORIA EN PERSONAS CON LESIÓN CEREBRAL ADQUIRIDA
}

\author{
APPROACHES AND FOUNDATIONS FOR A MODEL OF \\ AMBULATORY REHABILITATION IN PEOPLE WITH ACQUIRED \\ BRAIN INJURY
}

\begin{abstract}
Lorena Jiménez C. ${ }^{1 a}$, Christian Salas R. ${ }^{2 b}$, Marcos Maldonado D. ${ }^{1 a a}$, Alejandra Moya F. ${ }^{1 a}$, Soledad Lagos M. ${ }^{1 c}$, Carolina Herrera Ph. ${ }^{1 c}$, Daniela Gazzana S-A. ${ }^{1 d}$, Silvana Moreno ${ }^{1 a}$
\end{abstract}

\section{Resumen}

Introducción: Un porcentaje significativo de personas que adquieren una lesión cerebral necesita programas de rehabilitación ambulatoria. En Chile predomina aún un modelo biomédico de rehabilitación, no obstante, las nuevas guías clínicas recomiendan un abordaje interdisciplinario que considere las necesidades del paciente y su familia. Actualmente existe un vacío respecto a cómo operacionalizar intervenciones eficaces que respondan a este paradigma.

Objetivo: Encontrar evidencia que permita el desarrollo y evaluación de enfoques en neurorehabilitación ambulatoria de personas con lesión cerebral adquirida y las posibles metodologías de operacionalización de estos.

Metodología: Revisión inductiva y conceptual de la literatura.

Resultados: En la literatura chilena no existe evidencia empírica ni desarrollos sistemáticos sobre los fundamentos y la efectividad de los programas de rehabilitación ambulatoria para estas personas. En la literatura internacional se destacan tres enfoques: el enfoque holista, el centrado en el cliente y el basado en la planificación de metas. Si bien tales enfoques permiten guiar las acciones de los equipos, la evidencia de su efectividad, en base al criterio de expertos y pequeños estudios longitudinales y de caso único, es aun limitada. Conclusiones: Esencial a la construcción de identidad de un equipo de rehabilitación es determinar los principios y enfoques que sustente su labor. La complejidad y diversidad de los pacientes en la

\footnotetext{
Profesional Área Terapias Centro Ambulatorio de Clínica Los Coihues.

Estudiante de Doctorado en Neuropsicología, Universidad de Wales.

Kinesiólogo aa Kinesiólogo ${ }^{\text {b }}$ Psicólogo ${ }^{\mathrm{c}}$ Terapeuta Ocupacional.

d Fonoaudióloga, Contacto: mmaldonado@loscoihues.cl Fono: 56-2-230 6060.
} 
fase ambulatoria requiere que los equipos posean claros fundamentos para guiar la constante toma de decisiones. Es necesaria la evaluación de la eficacia de los programas de rehabilitación ambulatoria en Chile, desarrollando metodologías acordes a la naturaleza multifactorial del fenómeno.

\title{
Palabras Clave
}

Lesión Cerebral Adquirida, Enfoques y Modelos de Rehabilitación, Equipo Interdisciplinario.

\begin{abstract}
Introduction: A significant percentage of people who acquire a Brain Injury need outpatient rehabilitation programs. In Chile the rehabilitation field is in a transitional stage. Even though the biomedical model is predominant in rehabilitation, the clinical guidelines have begun to recommend an interdisciplinary approach that considers patients needs and their families. Now-a-days, there is a gap on how to operationalize effective interventions responding to this paradigm.

Objectives: To find evidence that sustains the development and assessment of outpatient neurorehabilitation approaches of people with brain injury and, to describe the possible operationalization methodologies of these. Methodology: Inductive and conceptual review of literature.

Results: In Chilean Literature does not exist empirical evidence nor systematic developments on the foundations and effectiveness of outpatient rehabilitation programs for people with brain injury. International Literature describes three approaches: holistic, client centered, and goal-based. While such approaches can guide the team actions, the evidence of their effectiveness, based on expert criteria and small longitudinal studies and single case studies is still limited.

Conclusions: For the development of a rehabilitation team identity, it is essential to determine the principles and approaches that support their work. Due to complexity and diversity of patients in the ambulatory phase, it is crucial that teams have clear foundations to guide the constant decision making. The evaluation of the effectiveness of ambulatory programs in Chile is a necessity; also it is to develop methodologies according to the nature of the phenomenon.
\end{abstract}

\section{Key Words}

Acquired brain injury, Approaches and Models of rehabilitation programs, Interdisciplinarity.

\section{INTRODUCCIÓN}

\section{La Lesión Cerebral Adquirida.}

Bajo el concepto de Lesión Cerebral Adquirida (LCA) se incluyen diversas patologías generadas por un daño cerebral agudo. Entre ellas se encuentran el Accidente Vascular Encefálico (ACV), el Trauma Ecéfalocraneano (TEC), el Daño Encefálico Post-quirúrgico, la Hemorragia Subaracnoídea, la Anoxia Cerebral, y otros insultos tóxicos, metabólicos, infecciosos o inflamatorios1. Las principales causas de LCA son el ACV $y$ el TEC $(1,2)$.

La OMS3, ubica al ACV en el segundo lugar de la carga global de enfermedad en mayores de 60 años. En Chile, a partir de los 20 años de edad, la tasa de mortalidad por ACV aumenta sostenidamente (3). La incidencia anual de los infartos cerebrales es aproximadamente 130 por cada cien mil habitantes, de los cuales la mitad 
muere o queda con invalidez, por lo que esta patología se ha convertido en la principal causa de discapacidad en el adulto4. Debido a razones epidemiológicas, y a la probada eficacia de ciertas intervenciones terapéuticas, el tratamiento del ACV fue incluido en el listado de patologías o condiciones de salud que forman parte del "Régimen de Garantías Explícitas en Salud" $(3,4)$.

Por su parte, en Chile, el TEC es la primera causa de muerte en la población de 20 a 40 años, y también es causa importante de secuelas neurológicas en pacientes de edad productiva (5).

Hemos señalado que un segmento relevante de la población que sobrevive a una LCA experimenta secuelas. Estas pueden ser de diversa índole (ver Tabla 1), impactando negativamente en un amplio espectro de habilidades adaptativas, como la capacidad de autocuidado, mantener relaciones sociales, tener éxito educacional o conseguir un trabajo (6).

Tabla 1:

Algunos de los Déficits que Resultan de una Lesión Cerebral Adquirida*

\begin{tabular}{|c|c|c|c|}
\hline \multicolumn{4}{|c|}{ Déficits que Resultan de la Lesión Cerebral Adquirida } \\
\hline Físicos & Comunicativos & Cognitivos & Conductual/Emocional \\
\hline $\begin{array}{c}\text { Parálisis o paresia } \\
\text { Tono muscular anormal } \\
\text { Ataxia/coordinación } \\
\text { - Déficits sensoriales } \\
\text { - Pérdida auditiva/visual } \\
\text { - Síntomas como cefalea, fatiga, } \\
\text { dolor, etc. } \\
\text { - Disfagia } \\
\text { - Convulsiones }\end{array}$ & $\begin{array}{l}\text { - Alt. Lenguaje Expre- } \\
\text { sivo } \\
\text { - Alt. Lenguaje Com- } \\
\text { prensivo } \\
\text { - Disartria } \\
\text { - Alexia } \\
\text { - Disfagia }\end{array}$ & $\begin{array}{l}\text { - Alt. Memoria } \\
\text { - Alt. Atencionales. } \\
\text { - Alt. Preceptúales. } \\
\text { - Alt. Resolución de } \\
\text { Problemas } \\
\text { - disminución del Insight } \\
\text { - Alt. de la conciencia de } \\
\text { peligro } \\
\text { - Déficit en Automonitoreo } \\
\text { - Alt. en el Juicio social }\end{array}$ & $\begin{array}{l}\text { - Labilidad emocional } \\
\text { - Disminución en la } \\
\text { iniciación de conductas } \\
\text { - Cambios de humor } \\
\text { - Problemas de ajuste } \\
\text { conductual a contexto } \\
\text { - Explosiones de ira } \\
\text { - Desinhibición } \\
\text { - Comportamiento sexual } \\
\text { inapropiado } \\
\text { - Baja motivación } \\
\text { - Psicosis }\end{array}$ \\
\hline
\end{tabular}

* Royal College of Physicians and British Society of Rehabilitation. Rehabilitation following acquired brain injury: national clinical guidelines. London; Turner-Stokes L, ed; 2003. (1).

El grado de impacto de estos déficits en la funcionalidad y participación depende de factores como la severidad de la lesión (extensión y localización), el perfil de los déficits (motor, cognitivo, conductual), la personalidad premórbida y la flexibilidad del ambiente familiar y social para adaptarse a los cambios y compensarlos (1). Al respecto, conviene recordar que los déficits impactan también en el sistema familiar, o las "otras víctimas" (7), quienes en el largo plazo pueden llegar a vivir bajo un considerable grado de estrés (8) y conflicto (9). Este es un punto de central relevancia en la etapa ambulatoria de la rehabilitación, y será abordado en detalle más adelante.
A nivel mundial, la rehabilitación de pacientes con LCA se ha desarrollado científicamente sólo en los últimos 50 años, en tanto que en nuestro país su evolución es reciente y experimental. La prevalencia de un modelo preponderantemente biomédico en las instituciones de rehabilitación, la inexistencia de una red pública de centros de rehabilitación especializados y la escasez de espacios de formación académica, son algunos de los factores que dan cuenta del desfase entre nuestra capacidad de disminuir la mortalidad de las víctimas de LCA y mejorar la calidad de vida de los sobrevivientes 10. 
Usualmente la evolución de los pacientes que adquieren una LCA se compone de una etapa sub-aguda de hospitalización (inpatient) y una fase ambulatoria (outpatient). Una vez de alta de las unidades de rehabilitación hospitalarias, los pacientes con lesiones moderadas o severas suelen continuar recibiendo terapia ambulatoria a una intensidad más baja. El principal objetivo de estos programas es la reintegración comunitaria11, variando en los objetivos específicos de acuerdo a las necesidades particulares de cada paciente.

Algunos países han desarrollado Guías Clínicas donde se incluyen recomendaciones basadas en diversos grados de evidencia científica y opinión de expertos respecto a la fase ambulatoria de reintegración comunitaria. Las actuales Guías Clínicas Chilenas de ACV, HSA y TEC, lamentablemente no definen de forma exhaustiva las intervenciones a realizar en esta etapa, señalando más bien amplios lineamientos; como el trabajo interdisciplinario de los equipos y la incorporación activa de los pacientes y sus familias1,4. Se hace necesario entonces, determinar los fundamentos de un programa de rehabilitación de LCA ambulatorio, así como describir las metodologías que permiten operacionalizar dichos fundamentos en intervenciones concretas y evaluables.

El propósito de este estudio es encontrar evidencia que permita el desarrollo y la evaluación de enfoques en la neurorehabilitación ambulatoria de personas con lesión cerebral adquirida y las posibles metodologías de operacionalización de estos.

\section{Metodo}

La presente revisión bibliográfica abarca el período comprendido entre enero de 1990 y diciembre de 2008. Se utilizaron tres bases de datos en línea: Medline, Dialnet y Scielo. En una primera selección los términos de búsqueda usados fueron: Brain Injury + [rehabilitation, outcome, ambulatory rehabilitation, team rehabilitation y family], posteriormente se agregaron: [goal planning y cognitive rehabilitation].

Luego de leer los resúmenes, en un segundo tamizaje se desecharon aquellos artículos que no abordaban el problema. Finalmente posterior a la lectura de los artículos en extenso, se revisaron e incluyeron algunos artículos y libros utilizados como referencias en los artículos seleccionados, cuidando que estos hubieran sido sometidos a un comité editorial. Además se consultaron seis guías clínicas Nacionales e Internacionales disponibles en línea, en relación al Daño Cerebral Adquirido.

\section{Resultados}

La siguiente tabla muestra los resultados de la búsqueda de referencias:

\begin{tabular}{|c|c|c|c|c|c|c|}
\hline \multirow{2}{*}{$\begin{array}{c}* \\
\text { Términos claves }\end{array}$} & \multicolumn{2}{|c|}{$\begin{array}{c}\text { Artículos encontrados por términos } \\
\text { claves }\end{array}$} & \multicolumn{3}{c|}{$\begin{array}{c}\text { Artículos seleccionados para la } \\
\text { revisión inductiva y conceptual* }\end{array}$} \\
\cline { 2 - 7 } & Pubmed & Dialnet & Scielo & Pubmed & Dialnet & Scielo \\
\hline Brain Injury + rehabilitation & 370 & 8 & 353 & 147 & 2 & 1 \\
\hline Brain Injury + outcome & 41 & 10 & 441 & 25 & 2 & 2 \\
\hline Brain Injury + team rehabilitation & 39 & 1 & 232 & 4 & 0 & 0 \\
\hline Brain Injury + family & 11 & 7 & 316 & 7 & 1 & 0 \\
\hline Brain Injury + goal planning & 53 & 0 & 140 & 7 & 0 & 0 \\
\hline Brain Injury + cognitive rehabilitation & 97 & 2 & 170 & 15 & 1 & 1 \\
\hline Brain Injury + ambulatory rehabilitation & 89 & 0 & 72 & 2 & 0 & 0 \\
\hline
\end{tabular}

* Todos estos artículos fueron seleccionados pero no todos incluidos en la presente revisión pues no tenían directa relación con el tema. 


\section{El Equipo de Trabajo en la}

\section{Neurorehabilitación Ambulatoria}

Como hemos señalado, en diversas guías clínicas se hace referencia a la intervención de equipos de rehabilitación multidisciplinarios $(12,13)$ e interdisciplinarios1, $(3,4)$.

El trabajo en equipo implica el desarrollo de un proceso, determinando la finalidad perseguida y las estrategias a utilizar. Aspectos como la comunicación y cooperación con otros profesionales, la integración del paciente y su familia, la flexibilidad, la distribución de responsabilidades y de poder, se desarrollan en grado variable, dependiendo de la estructura del equipo. En función de lo anterior, se demandará de los integrantes del equipo ciertas habilidades técnicas y sociales que sustenten y faciliten, el logro de los objetivos planteados.

En la literatura del ámbito de la salud se mencionan tres sistemas de equipo de trabajo: multidisciplinario, interdisciplinario y transdisciplinario $(14,15,16)$. La diferencia fundamental entre ellos radica en el grado en que los profesionales traspasan los límites de cada especialidad16 (Para una descripción detallada de cada sistema de organización de equipo, ver tabla 2).

Tabla 2:

Tabla Comparativa de los Modelos de Trabajo en Equipo *

\begin{tabular}{|c|c|c|c|}
\hline \multirow{2}{*}{ Variables Metodológicas } & \multicolumn{3}{|c|}{ Modelos de Trabajo en Equipo en Salud } \\
\hline & Multidisciplinario & Interdisciplinario & Transdisciplinario \\
\hline $\begin{array}{l}\text { Ámbito de cada experto en } \\
\text { el abordaje terapéutico. }\end{array}$ & Circunscrito & Circunscrito, coordinado & $\begin{array}{l}\text { Coordinado y ampliado por un entrena- } \\
\text { miento cruzado en el quehacer de las } \\
\text { otras disciplinas }\end{array}$ \\
\hline $\begin{array}{l}\text { Intercambio de informa- } \\
\text { ción sobre el paciente. }\end{array}$ & Escasa y general & $\begin{array}{l}\text { Con fines colaborativos y } \\
\text { transversales }\end{array}$ & $\begin{array}{c}\text { Completa, para un abordaje transversal } \\
\text { orientado a la meta. }\end{array}$ \\
\hline $\begin{array}{c}\text { Quién establece las Metas } \\
\text { de trabajo. }\end{array}$ & El líder o el equipo & $\begin{array}{l}\text { El equipo, que puede in- } \\
\text { cluir al paciente/familia }\end{array}$ & $\begin{array}{l}\text { El equipo, que incluye al paciente/fa- } \\
\text { milia }\end{array}$ \\
\hline Tipo de Metas de Trabajo. & Independientes & $\begin{array}{c}\text { Coordinadas, colaborativas } \\
\text { y holistas }\end{array}$ & $\begin{array}{l}\text { Transversales a todas las disciplinas in- } \\
\text { volucradas, } \\
\text { holistas (incluye a la familia) }\end{array}$ \\
\hline $\begin{array}{l}\text { Coordinación de Especia- } \\
\text { lidades. }\end{array}$ & No & $\mathrm{Si}$ & $\mathrm{Si}$ \\
\hline Toma de Decisiones & Independiente & Coordinadas & Consensuadas \\
\hline $\begin{array}{l}\text { Recursos de los Profesio- } \\
\text { nales Involucrados. }\end{array}$ & $\begin{array}{l}\text { Experticia en su disci- } \\
\text { plina }\end{array}$ & $\begin{array}{l}\text { - Experticia en su disciplina } \\
\text { - Tiempo } \\
\text { - Compromiso } \\
\text { - Comunicación } \\
\text { - Confianza mutua } \\
\text { - Adquisición de una meto- } \\
\text { dología de trabajo } \\
\text { - Liderazgo }\end{array}$ & $\begin{array}{c}\text { - Experticia y experiencia en su disci- } \\
\text { plina } \\
\text { - Tiempo } \\
\text { - Compromiso } \\
\text { - Comunicación } \\
\text { - Confianza mutua } \\
\text { - Adquisición de una metodología de } \\
\text { trabajo } \\
\text { - Alto grado de organización y liderazgo. }\end{array}$ \\
\hline
\end{tabular}




\begin{tabular}{|c|c|c|c|}
\hline \multirow{2}{*}{ Variables Metodológicas } & \multicolumn{3}{|c|}{ Modelos de Trabajo en Equipo en Salud } \\
\hline & Multidisciplinario & Interdisciplinario & Transdisciplinario \\
\hline $\begin{array}{c}\text { Recursos Institucionales } \\
\text { Necesarios }\end{array}$ & Los de cada disciplina & $\begin{array}{l}\text { - Los de cada disciplina } \\
\text { - Hrs. de reunión de } \\
\text { equipo para establecimien- } \\
\text { to y seguimiento de metas } \\
\text { y coordinación de } \\
\text { intervenciones } \\
\text { - Hrs. de coordinación } \\
\text { - Sala de reunión y material } \\
\text { de oficina }\end{array}$ & $\begin{array}{l}\text { - Los de cada disciplina } \\
\text { - Hrs. de reunión de equipo para es- } \\
\text { tablecimiento y seguimiento de metas } \\
\text { conjuntas, distribución de tareas y fertili- } \\
\text { zación cruzada de cada disciplina } \\
\text { - Hrs. de coordinación } \\
\text { - Sala de reunión y material de oficina }\end{array}$ \\
\hline
\end{tabular}

* Bibliografía: ROY S.A. The process of reorganization. In H.G. Garner (Ed), Team model and experience in education. Boston: Ally \& Bacon; 1995. p. 85-101.

DYER, JEAN A. Multidisciplinary, Interdisciplinary, and Transdisciplinary Educational Model and Nursing Education. Nursing Education Perspectives 2003; 24, 4: 186 -188.

MAGILL-EVANS J, HODGE M, DARRAH J. Establishing a Transdisciplinary Research Team in Academia. Journal of Allied Health 2002; $31 \mathrm{n}^{\circ} 4$ : 222-226.

\section{Enfoques en la Rehabilitación Ambulatoria de Personas con LCA}

Los programas de rehabilitación ambulatoria implican un alto costo debido al carácter personalizado de sus intervenciones. Cullen y cols11 llevaron a cabo una revisión de la literatura sobre la eficacia de diversos programas de rehabilitación de LCA desde 1980 a 2005. Como principales hallazgos respecto a la fase ambulatoria se determinó la existencia de: evidencia limitada de que una rehabilitación social y conductual de base comunitaria, de al menos 6 meses, resulta en mayores grados de independencia y de actividad social y menor necesidad de cuidado; evidencia moderada de que el involucramiento directo del paciente en el establecimiento de metas de rehabilitación resulta en una mejora significativa del logro de objetivos, manteniéndose los resultados incluso dos meses después del alta; y evidencia limitada de que la participación en programas ambulatorios reduce las alteraciones de conciencia de enfermedad y malestar, mejorando la participación social incluso a un año de alta' .

1 La evidencia fue clasificada como a) Sólida: si era apoyada al menos por dos ensayos aleatorios controlados de buena calidad. b) Moderada: apoyada por un ensayo aleatorio controlado de buena calidad. c) Limitada:
La realidad de nuestro quehacer como equipo de rehabilitación de un centro ambulatorio compuesto por kinesiólogos, terapeutas ocupacionales, fonoaudiólogos, neuropsicólogos y fisiatras, nos ha llevado inevitablemente a adherir a ciertos enfoques que configuran tanto la estructura de los procesos (enfoque centrado en el cliente, enfoque de planificación de metas) como su comprensión (enfoque holista). En esta línea de análisis presentamos la descripción de tres enfoques teóricos que han penetrado profundamente el quehacer de nuestro equipo, transformándose en faros que permiten no extraviarse en la cotidiana y compleja toma de decisiones.

Si bien existen diversos tipos de programas ambulatorios (ver tabla 3), de acuerdo al tipo de paciente y a los objetivos de rehabilitación, existen ciertos principios generales a los cuales estos programas adhieren en mayor o menor grado. Para los fines de esta revisión los hemos denominado enfoques.

apoyada por otros tipos de estudio no experimentales, ensayos controlados prospectivos o retrospectivos, intervenciones en grupo único. d) Opinión de expertos. e) Evidencia conflictiva. 
Tabla 3:

Programas de Rehabilitación Post Agudos de Lesión Cerebral*

\begin{tabular}{|c|c|c|c|}
\hline Tipo & Objetivos Principales & $\begin{array}{c}\text { Características de los } \\
\text { pacientes }\end{array}$ & $\begin{array}{c}\text { Principales componentes del } \\
\text { programa }\end{array}$ \\
\hline $\begin{array}{l}\text { Ambulatoria } \\
\text { Tradicional }\end{array}$ & Autocuidado y AVDs & Daño leve a Severo & $\begin{array}{c}\text { Kinesioterapia, Terapia Ocupacional } \\
\text { y Fonoaudiología }\end{array}$ \\
\hline $\begin{array}{l}\text { Reinserción } \\
\text { Comunitaria }\end{array}$ & $\begin{array}{l}\text { Independencia en hogar, co- } \\
\text { munidad y regreso al trabajo }\end{array}$ & Daño leve a moderado & $\begin{array}{c}\text { Terapias de enfoque comunitario con } \\
\text { énfasis vocacional }\end{array}$ \\
\hline Holista & $\begin{array}{c}\text { Adaptación psicosocial, com- } \\
\text { pensación de alteraciones cog- } \\
\text { nitivas, retorno al trabajo }\end{array}$ & $\begin{array}{c}\text { Daño leve a moderado. } \\
\text { Alteración de la conciencia } \\
\text { de enfermedad. }\end{array}$ & $\begin{array}{l}\text { Ambiente Terapéutico. Orientación } \\
\text { Neuropsicológica. Equipo Integrado. }\end{array}$ \\
\hline Residencial & $\begin{array}{l}\text { AVDs e independencia en el } \\
\text { hogar }\end{array}$ & Daño moderado a Severo & $\begin{array}{l}\text { Entrenamiento en habilidades } \\
\text { funcionales en ambiente residencial. }\end{array}$ \\
\hline Conductual & $\begin{array}{l}\text { Control y estabilidad } \\
\text { conductual. }\end{array}$ & $\begin{array}{l}\text { Trastornos neuropsiquiátri- } \\
\text { cos y neuroconductuales }\end{array}$ & $\begin{array}{l}\text { Modificación conductual en } \\
\text { ambientes estructurados }\end{array}$ \\
\hline LifeLong Living & Calidad de vida & Daño moderado a severo & $\begin{array}{c}\text { Integración interpersonal y } \\
\text { comunitaria. }\end{array}$ \\
\hline
\end{tabular}

* Bibliografía: TREXLER L. Empirical support for Neuropsychological Rehabilitation. In International handbook of Neuropsychological Rehabilitation. Christensen \& Uzzel eds. Kluwer Academic/ Plenum Publishers. New York, USA; 2000. p. 137-150.

\section{a.- Enfoque Centrado en el Cliente}

Hasta los años 60, los médicos y profesionales expertos determinaban lo que ellos consideraban que era "mejor" para el paciente. En los 70s, la emergencia de políticas de consumo abrió la posibilidad de considerar a la persona con discapacidad en un rol más activo en la toma de decisiones. Actualmente, la práctica basada en la evidencia ha generado una involución al respecto, debido a que las decisiones son tomadas en base a criterios clínicos, fundados en investigaciones sistemáticas (17). No obstante, si revisamos los paradigmas actuales de los centros emblemáticos de rehabilitación ambulatoria, es observable un movimiento hacia la mutualidad en la toma de decisiones y la distribución del poder. Algo sensato, considerando que un elemento central de todo proceso ambulatorio, implica facilitar retomar el control y autonomía sobre la vida.
Respecto a los factores que permiten desarrollar un enfoque centrado en el cliente, Cott (17) indagó en la percepción de 33 pacientes con enfermedades crónicas y discapacidad, quienes señalaron como relevante: a) la individualización de los programas de acuerdo a las necesidades de cada paciente en orden a prepararlos para la vida en el mundo real; b) la participación conjunta con los profesionales en el establecimiento de metas y toma de decisiones; c) la obtención de resultados significativos para el paciente; d) compartir con el paciente la información y educación adecuada de acuerdo al momento de evolución y deseos del paciente; e) el apoyo emocional; f) el involucramiento de familiares y pares en el proceso de rehabilitación; f) la coordinación y continuidad a través de los servicios sociales y de salud. 
En relación al posible impacto del planteamiento conjunto de objetivos en el proceso de rehabilitación, Holliday y cols18 a pesar de que no observaron diferencias en logros funcionales entre el grupo experimental y el grupo control, así como tampoco en la duración de la estadía en el programa; sí encontraron que el grupo de pacientes que participó en la determinación y supervisión activa de sus objetivos: estableció más objetivos relativos a la participación, calificó sus objetivos como más relevantes y, expresó mayor satisfacción con su proceso de rehabilitación.

\section{b.- Enfoque Holista de la Neuro-Rehabilitación}

Históricamente en la rehabilitación de sobrevivientes de LCA se ha enfatizado la reparación de los déficits físicos y cognitivos (Enfoque Tradicional). Actualmente, tal visión es considerada como estrecha e insuficiente, desplazando el énfasis hacia los problemas particulares que estos déficits causan en la vida de los sobrevivientes (19). Por ejemplo, no poder vestirse u organizar mentalmente una actividad, y cómo esto impacta en la participación social y laboral (20). Este enfoque se ha denominado Contextualizado (19) u Holista (21).

Desde una perspectiva Holista, la rehabilitación es definida como un proceso que busca aumentar tanto la funcionalidad física como la capacidad de procesar información (Ben-Yishay \& Prigatano, 1990 citado en Wilson) (22), disminuyendo problemas asociados como la depresión, ansiedad, dificultades de comunicación e interacción social (22). Esto requiere la aplicación de procedimientos, técnicas y utilización de apoyos para retomar de forma segura, productiva e independiente las tareas de la vida diaria6. Tal proceso es bidireccional e interactivo, involucra a la persona con discapacidad, el equipo terapéutico, la familia y miembros de la comunidad (McLellan, 1991 citado en Wilson) (22).

Una rehabilitación holista exige la comprensión de la compleja interacción entre los ámbitos físico, cognitivo, conductual y afectivo, dentro de un contexto centrado en las necesidades del paciente y su familia (10). Prigatano (23) destaca ciertos ingredientes básicos de este enfoque que deberían ser considerados en cualquier modelo comprensivo de rehabilitación de personas con LCA: a) la rehabilitación cognitiva y las intervenciones psicoterapéuticas; b) el establecimiento de un ambiente que facilite la recuperación, la adaptación y evite el deterioro; c) el establecimiento de un ambiente laboral y educacional protegido; d) el compromiso activo de los miembros de la familia en el proceso de rehabilitación y; e) el manejo profesional de las reacciones emocionales positivas y negativas de los terapeutas hacia el paciente con LCA y su familia.

En relación a la efectividad de los programas de rehabilitación holistas, la evidencia disponible es discreta, pero favorable. Algunos estudios dan cuenta de una mayor reintegración laboral y de actividades productivas $(25,26,27,28)$. También se han encontrado mejoras significativas en la capacidad de cohabitación y autonomía en el hogar $(29,30,28)$, la cantidad y calidad de actividades de recreación, y la disminución del número de visitas hospitalarias y prestaciones terapéuticas (30). En el índice de discapacidad física del Portland Adaptability Inventory se detectaron mejorías significativas (29), al igual que en las alteraciones de conciencia de sí, nivel de estrés (31), alteraciones cognitivas, déficits en habilidades sociales, control emocional y conductual (28).

\section{c.- Enfoque en Base a Planificación de Metas}

La adecuada planificación de los objetivos, medios y timming de un proceso de rehabilitación es el núcleo de las intervenciones, permitiendo construir una colaboración realista entre equipo y paciente/familia. Esto implica dejar atrás el modelo tradicional donde el terapeuta es el experto y el paciente alguien pasivo sobre quien se interviene. Un abordaje consecuente al modelo holista exige, por el contrario, la planificación conjunta de metas realistas, desafiantes y significativas en la vida cotidiana del paciente (22). En esta línea, la planificación de metas permite considerar como objeto de intervención la discapacidad y los problemas de participación; niveles de análisis que, a diferencia 
del déficit, refieren más bien a la calidad de vida, son multifactoriales y poseen una naturaleza subjetiva (32).

La planificación de metas ha sido utilizada ya por muchos años en diversos ambientes de rehabilitación, con diversos grados de sofisticación y resultados positivos en poblaciones como: parálisis cerebral (33), adultos con LCA $(34,35,36)$ pacientes psiquiátricos (37), personas con lesión medular (38) y problemas de aprendizaje (39).

La Operacionalización del enfoque centrado en la planificación de metas involucra: a) la designación de un encargado del caso que canalice la información entre la familia y el equipo; b) la formulación de un plan de evaluación diseñado en conjunto por los especialistas para construir un perfil de déficits, motivaciones y recursos; c) reuniones sistemáticas de planificación de metas entre el equipo, el paciente y la familia; d) el diseño de una lista de problemas construida colaborativamente entre el equipo y el paciente/familia; e) el establecimiento de planes de acción que registren el logro de las metas, y en caso de no lograrlas, por qué (22).

La planificación de metas no es una acción exclusiva a las primeras etapas del proceso de rehabilitación. Por el contrario, posee un carácter nuclear, ocurriendo a lo largo de todo el proceso por medio de la constante revision de logros y replanteamiento de objetivos. Es incluso posible afirmar que la planificacion de metas es una intervención en sí misma, ya que facilita la constante regulación de expectativas entre actores del proceso, promueve la adherencia al tratamiento, explicita el para qué se trabaja y fomenta una mayor conciencia sobre los deficits.

\section{Factores del Paciente en la Rehabilitación Ambulatoria}

Hasta aquí hemos desarrollado tres enfoques que articulan un proceso de rehabilitación ambulatoria integral de sobrevivientes de LCA: la centralidad en el cliente, la comprensión holista de sus problemas y la necesidad de co-construir las metas del proceso. A continuación señalaremos otros factores, propios del paciente y su familia, los cuales si bien no configuran enfoques por sí solos, son destacados por la literatura como relevantes para el éxito del proceso de rehabilitación.

\section{a.- Los Tiempos del Proceso}

La evolución de las áreas motoras, cognitivas, afectivas y conductuales del paciente durante la fase ambulatoria no suele poseer un desarrollo homogéneo. Por ejemplo, la recuperacion de la capacidad de marcha puede ocurrir mucho antes que la capacidad de realizar multitarea. Se hace entonces relevante determinar el perfil particular de evolución de las diversas áreas en cada paciente, fortaleciendo aquellas que han mostrado avances significativos y diseñando intervenciones específicas para las áreas rezagadas. La facilitación del proceso de transición al hogar será el objetivo transversal que guíe todas las intervenciones del equipo.

\section{b.- Las Expectativas}

El problema de las expectativas del paciente, grupo familiar y equipo, es un punto crucial para estructurar los objetivos e intervenciones del tratamiento. Muchos procesos de rehabilitación sucumben al no lograr abordar las expectativas con experticia. Se han descrito, por ejemplo, interacciones de culpabilización mutua y odio entre el equipo, el paciente y su familia, al no cumplir las expectativas del tratamiento (40). Por el contrario, el mantenimiento de una buena alianza entre los actores se relaciona con resultados terapéuticos positivos $(41,27)$, facilitando la adecuación de las expectativas y la generalización del aprendizaje fuera de ambiente terapéutico, un punto crucial en la fase ambulatoria.

\section{c.- La Participación Activa de la Familia}

La generación de modelos de colaboración con las familias de sobrevivientes es un desafío de suma importancia en la fase ambulatoria (42). Debido a las limitaciones físicas, cognitivas y conductuales, el gru- 
po familiar se configura en un núcleo estratégico de intervención, siendo la red de apoyo más potente y eficaz en el ajuste de la persona con LCA a la vida social (43).

La literatura identifica la importancia del involucramiento familiar en el éxito de la rehabilitación de personas con LCA (44). Algunos estudios han mostrado que el involucramiento efectivo de las familias se relaciona con mejorías funcionales (45) y de adaptación46 en pacientes con ACV. Por el contrario, altos niveles de desacuerdo entre la familia y el equipo, respecto al funcionamiento del paciente, se asocia con una escasa alianza de trabajo y menor colaboración en el proceso de rehabilitación (47).

\section{d.- Conciencia de Enfermedad, Negación y Duelo}

Existe una serie de factores cognitivo-afectivos que inciden en la colaboración de los pacientes, disminuyendo la efectividad de la rehabilitación $(49,50)$ y exigiendo a los equipos diseñar intervenciones específicas (51). La conciencia de enfermedad ha sido definida como la capacidad de comprender la naturaleza y efectos de los déficits, incluyendo la necesidad de rehabilitación48. Los déficits en la conciencia de enfermedad, denominados también como anosognosia, son altamente frecuentes luego de un TEC (52) y ACV del hemisferio derecho o de los lóbulos frontales (53). Por otro lado, la negación, como fenómeno propio del proceso de duelo, presenta similitudes con las fallas de conciencia de enfermedad, instalando también importantes dificultades en los procesos (54). Ambos factores exigen al equipo evaluar acuciosamente la presencia y alcance de estas alteraciones, dando especial énfasis a la significación individual de lo perdido. Sólo de esta manera es posible comprender qué es lo que el paciente busca recuperar y determinar así cómo ayudarlo.

\section{DISCUSIÓN}

Diseñar intervenciones que aborden las múltiples interacciones entre los ámbitos físico, cognitivo, conductual, afectivo y familiar, exige equipos capacitados en resolver problemas de alta complejidad. Es por esto que la estructura y dinámica del equipo de rehabilitación se constituye en un factor altamente determinante respecto a la efectividad de las intervenciones. En nuestra experiencia lo que suele suceder, respecto al estilo de funcionamiento del equipo de neurorehabilitación, es que este se desplaza en un continuo desde lo multidisciplinario a lo transdisciplinario, de acuerdo a las necesidades y disponibilidad de recursos económicos, técnicos y humanos. Debido al concepto actual de rehabilitación que los centros utilizan, y a las políticas administrativas que sustentan su quehacer, son pocos los equipos que funcionan en una manera verdaderamente transdisciplinaria (16). Nuestra dinámica se enmarca predominantemente en lo interdisciplinario.

Una tarea central, en la construcción de la identidad de un equipo de neurorehabilitación ambulatoria es determinar los principios y enfoques que sustentan su labor cotidiana. Sólo mediante la internalización de dichos fundamentos el equipo puede enfrentar adecuadamente los múltiples escenarios que instalan cada paciente y familia. El enfoque centrado en el cliente y la perspectiva holista de la rehabilitación ofrecen lineamientos generales para comprender el fenómeno de la rehabilitación. El enfoque centrado en la planificación de metas ofrece una estructura que permite desenvolver e intervenir sobre el proceso. En nuestra experiencia, la articulación de estos tres enfoques ha facilitado el involucramiento activo de los pacientes y sus familias, permitiendo no sólo aumentar la comprensión de para qué se trabaja, sino también intervenir sobre las alteraciones en la conciencia de déficits.

La planificación de metas merece una reflexión aparte. Esta no debe ser considerada como una etapa inicial del proceso de rehabilitación, sino más bien como el núcleo de todo el proceso. Mediante la determinación y evaluación constante de los objetivos el equipo logra 
monitorear el nivel de adherencia de los pacientes y familias, regular las expectativas y acceder a las valoraciones de los pacientes respecto a qué es aquello significativo a lograr. Por otro lado, la planificación de metas permite salvar las dificultades metodológicas que implica medir la eficacia de intervenciones que apuntan a problemas de participación, donde la significación individual de los objetivos, y la percepción subjetiva de su logro son los criterios centrales. Si consideramos, además, que los procesos de rehabilitación son de alto costo, es una tarea central poder ofrecer evidencia a las instituciones que financian los tratamientos. La planificación de metas permite ofrecer indicadores claros y objetivos de los procesos, sin abandonar la cualidad subjetiva de los problemas.

\section{ConClusion}

Como hemos señalado en el artículo, el estado de la neurorehabilitación en Chile es incipiente, por lo cual consideramos como un primer paso valioso determinar los posibles fundamentos de un modelo de abordaje en esta población. Este es sólo el primer paso y son numerosas las tareas pendientes. Algunas líneas de trabajo a considerar en el futuro son: a) la efectividad de los programas en términos de calidad de vida, estrés del cuidador y nivel de reinserción comunitaria; b) la efectividad de las intervenciones de acuerdo a las diversas subpoblaciones que presentan una LCA; c) las ventajas de un abordaje holista por sobre uno tradicional; d) las ventajas de un abordaje transdisciplinario por sobre uno interdisciplinario; e) el sostenimiento de los logros en el tiempo una vez abandonado el centro.

\section{Agradecimientos}

Médico Fisiatra Tania Gutiérrez P. por compartir y apoyar la visión que guía este trabajo y sus invaluables sugerencias y correcciones al manuscrito. Psicóloga Pamela Santibañez, por la revisión y aportes al manuscrito final.

\section{RefERENCIAS BibliOGRAfICAS}

(1) Royal College of Physicians and British Society of Rehabilitation (RCP and BSRM). Rehabilitation following acquired brain injury: national clinical guidelines. London; Turner-Stokes L, Ed; 2003.

(2) Ministerio de Trabajo y Asuntos Sociales Secretaría de Estado de Servicios Sociales, Familias y Discapacidad Instituto de Mayores y Servicios Sociales (IMSERSO). Modelo de atención a las personas con daño cerebral. España. Disponible en: http://www. seg-social.es/imserso/documentacion/pubcat. Consultado en Junio 2008.

(3) Republica de Chile, Ministerio de Salud (MINSAL). Guía Clínica, ACV isquémico del adulto 2006. Disponible en: http://www.minsal.cl/ici/guias_clinicas_ges2006/ACV_GCAUGE_2006_version62.pdf. Consultado en Noviembre 2007.

(4) Republica de Chile, Ministerio de Salud (MINSAL). Clínica Ataque Cerebrovascular Isquémico del Adulto. Septiembre 2007. Disponible en: http:// www.redsalud.gov.cl/archivos/guiasges/isquemico. pdf. Consultado en Junio 2008.

(5) Republica de Chile, Ministerio de Salud (MINSAL). Guía Clínica, Atención de Urgencia del Traumatismo Craneocefálico 2007. Disponible en: http:// www.minsal.cl/ici/reforma_de_salud/GPCGesTEC-2007.pdf. Consultado en Noviembre 2007.

(6) MATEER C. Introducción a la rehabilitación cognitiva. Avances en Psicología Clínica Latinoamericana 2003; 21: 11-20.

(7) FLORIAN V, KATZ S. The other victims of traumatic brain injury: Consequences for family members. Neuropsychology 1991; 5: 267-279.

(8) MARSH N, KERSEL D, HAVILL J, SLEIGH J. Caregiver burden at 1 year following severe traumatic brain injury. Brain Injury 1998; 12: Issue 12: 1045-1059.

(9) MORIS, M. Consideraciones psicológicas para una intervención grupal en familias que conviven con secuelas de un daño cerebral adquirido. Revista Gaceta Universitaria 2005; 1; 2; 197-201. 
(10) SALAS C, BAEZA M, GARREAUD M, DACCARETT C. Experiencias y desafíos en rehabilitación cognitiva: ¿Hacia un modelo de intervención contextualizado? Revista Chilena de Neuropsicología, 2007; 2: $21-30$.

(11) CULLEN N, CHUNDAMALA J, BAYLEY M, JUTAI J. The efficacy of acquired brain injury rehabilitation. Brain Injury 2007; 2: Issue 2: 113-132

(12) Stroke Foundation New Zealand (SFNZ). Life after stroke, New Zealand guideline for management of stroke. Wellington; Stroke Foundation New Zealand Inc.; 2003.

(13) Republica de Chile, Ministerio de Salud (MINSAL). Guía Clínica, Hemorragia Subaracnoidea Secundaria a Rotura de Aneurismas Cerebrales 2007. Disponible en: http://www.minsal.cl/ici/reforma_ de_salud/GPCGes-HSA-2007.pdf. Consultado en: Noviembre 2007.

(14) ROY S.A. The process of reorganization. In H.G. Garner Ed, Team model and experience in education. Boston: Ally \& Bacon; 1995. p. 85-101.

(15) DYER J. Multidisciplinary, Interdisciplinary, and Transdisciplinary Educational Model and Nursing Education. Nursing Education Perspectives 2003; 24, 4: 186 -188.

(16) MAGILL-EVANS J, HODGE M, DARRAH J. Establishing a Transdisciplinary Research Team in Academia. Journal of Allied Health 2002; $31 \mathrm{n}^{\circ}$ 4: 222226.

(17) COTT C. Client-centred rehabilitation: client perspectives. Disability and rehabilitation 2004; $26 \mathrm{n}^{\circ}$ 24: 1411-1422

(18) HOLLIDAY RC, CANO S, FREEMAN JA, PLAYFORD ED. Should patients participate in clinical decision making? An optimised balance block design controlled study of goal setting in a rehabilitation unit. Journal of Neurology, Neurosurgery and Psychiatry 2007; 78: 576-580.

(19) YLVISAKER M, HANKS R, JOHNSON- GREENE D. Perspectives on rehabilitation of individual with cognitive impairment after brain injury: Rationale for reconsideration of theoretical paradigms. The journal of head and trauma rehabilitation 2002; 17:3: 191-209.

(20) WILSON B. Cognitive Rehabilitation: How it is and how it might be. Journal of the International Neuropsychological Society 1997; 3: 487 - 496.

(21) BEN-YISHAY Y. Postacute Neuropsychological Rehabilitation. A Holistic Perspective. En International Handbook of neuropsychological rehabilitation, edited by Christensen, A-L \& Uzzell, B. P. Kluwer Academic/ Plenum Publishers. New York, USA; 2000. p. 127-135.

(22) WILSON B, EVANS J, KEOHANE C. Cognitive Rehabilitation: A Goal-Planning Approach. Journal of Head and Trauma Rehabilitation 2002; 17, 6: 542

(23) PRIGATANO GP. "Principles of Neuropsychological Rehabilitation". New York: Oxford University Press. 1999.

(24) TREXLER L. Empirical support for Neuropsychological Rehabilitation. In International handbook of Neuropsychological Rehabilitation. Christensen \& Uzzel Eds. Kluwer Academic/ Plenum Publishers. New York, USA; 2000. p. 137-150.

(25) PRIGATANO GP, FORDYCE DJ, ZEINER HK, ROUECHE JR, PEPPING M, WORD BC. Neuropscyhological rehabilitation after closed head injury in young adults. J Neurol Neurosurgery and Psychiatry 1984; 47: 505-513.

(26) BEN-YISHAI Y, SILVER SM, PIASETSKY E, RATTOCK J. Relationship between employability and vocational outcome after intensive holistic cognitive rehabilitation. Journal of Head Trauma Rehabilitation 1987; 2: (1): 35-48.

(27) PRIGATANO GP, KLONOFF PS, O'BRYEN KP, ALTMAN IM, AMIN K, CHIAPELLO D, y cols. Productivity after neuropsychologically oriented millieu rehabilitation. J Head Trauma 1994; 9: 91-102

(28) MALEC J. Impact of comprehensive day treatment on societal participation for persons with acquired brain injury. Arch Phys Med Rehabil 2001; 82: Issue 7: 885-895.

(29) MALEC JF, SMIEGELSKI JS, DEPOMPOLO RW, THOMPSON JM. Outcome evaluation and predic- 
tion in a comprehensive integrated post acute outpatient brain injury rehabilitation program. Brain injury 1993; 7: 15-29.

(30) CHRISTENSEN AL, PINNER EM, MOLLER-PEDERSEN P, TEASDALE TW, TREXLER LE. Psychosocial outcome following individualized neuropsychological rehabilitation of brain damage. Acta Neurological Scandanavia 1992; 85(1): 32-38.

(31) MALEC J, MOESSNER A. Self-awareness, distress, and postacute rehabilitation outcome. Rehabilitation Psychology 2000; 45 (3): 227-241.

(32) MCMILLIAN TM, SPARKES C. Goal Planning and Neurorehabilitation: The Wolfson Neurorehabilitation Center Appoach. Neuropsychological Rehabilitation 1999; 9 n 3-4: 241-51.

(33) BOWYER E, MCLELLAN DL, ARNEY J, CAMPBELL $M J$. A randomised control trial of different intensities of physiotherapy and different goal setting procedures in 44 children with cerebral palsy. Developmental Medicine and Child Neurology 1996; 38: 226-237.

(34) WARD C, MCINTOSH S. The rehabilitation process, a neurological perspective In R.J. Greenwood, M. Barnes, T.M. MaMillan, \& C.D. Ward, Eds, Neurological rehabilitation. Hove, UK: Psychology Press. 1993. p. 13-28.

(35) WEBB PM, GLUECKHAUF RL. The effects of direct involvement in goal setting on rehabilitation outcome for persons with traumatic brain injuries. Rehabilitation psychology 1994; 39: 179-188.

(36) MCGRATH JR, MARKS JA, DAVIS AM. Towards interdisciplinary rehabilitation: further developments at Rivermead rehabilitation centre. Clinical rehabilitation 1995; 9 n4: 320-326.

(37) ROTHERY D, WRATE R, MCCABE R, ASPIN J, BRYCE G. Treatment goal- planning: Outcome findings of a British prospective multi-centre study of adolescent inpatient units. European Child and Adolescent Psychiatry 1995; vol. 4: n³: 209-220.

(38) MACLEOD GM, MACLEOD L. Evaluation of client and staff satisfaction with a Goal Planning project implemented with people with spinal injuries. Spinal Cord 1996; 34(9): 525-530.

(39) STURMEY P. Goal planning for adults with a mental handicap. Outcome research staff training and management. Mental handicap research 1995; 5, 92-108.

(40) GANS JS. Hate in the rehabilitation setting. Arch Phys Med Rehabil 1983; 64 (4): 176-179.

(41) KLONOFF P, LAMB D, HENDERSON S, SHEPPERD J. Outcome assessment after milieu-oriented neurorehabilitation: New considerations. Arch Phys Med Rehabil 1998; 79: Issue 6: 684-6902.

(42) MOORE M, MCLAUGHLIN A, TODIS B, LARSEN J, GLANG A. What does it Take to Collaborate with Families Affected by Brain Injury? A Preliminary Model. The Journal of Head Trauma Rehabilitation 2001; 16 (5): 498-511.

(43) LOURO I. Matriz de Salud del Grupo Familiar: Un Recurso para el Diagnóstico de la Situación de Salud de la Familia. Revista Cubana de Medicina General Integral 2004; 20: No 3.

(44) SANDER A,CAROSELLI J, HIGH W, BECKER C, NEESE L, SCHEIBEL R. Relationship of family functioning to progress in a post acute rehabilitation programme following traumatic brain injury, Brain Injury 2002; 16: 649-657.

(45) DEJONG G, BRANCH LG. Predicting the stroke patient's ability. Stroke 1982; 13: 648-655.

(46) BAKER AC. The spouse's positive effect on the stroke patient's recovery. Rehabilitation Nursing 1993; 18: 30-33.

(47) SHERER M, EVANS C, LEVERENZ J, STOUTER J, IRBY J, LEE J y cols. Therapeutic alliance in postacute brain injury rehabilitation: Predictors of strenght of alliance and impact of alliance on outcome. Brain Injury 2007; 21 (7): 663-672.

(48) COETZER R. Grief, Self-Awareness and psychotherapy following brain injury. Illness, crisis and Loss 2004; 12 n²: 171-186.

(49) GIALANELLA B, MONGUUZZI V, SANTORO R, ROCCJI S. Functional recovery after hemiplegia in 
patients with neglect the rehabilitative role of anosognosia. Stroke 2005; 36: 2678-90.

(50) PEDERSEN P, JORGENSEN $\mathrm{H}$, NAKAYAMA $\mathrm{H}$, RAASCHOU $\mathrm{H}$, OLSEN T. Frequency, determinants and consequences of anosognosia in acute stroke. J Neurol Rehab 1996; 10: 243-250.

(51) FLEMING J, OWNSWORTH T. A review of awareness interventions in brain injury rehabilitation. Neuropsychological Rehabilitation 2006; 16 (4): 474-500.

(52) PRIGATANO G. Disturbances of self-awareness and rehabilitation of patients with traumatic brain injury. A 20-year perspective. Journal of Head and Trauma Rehabilitation 2005; 20(1): 19-29.

(53) ORFEI M, ROBINSON R, PRIGATANO G, STARKSTEIN S, RUSCH N, BRIA P, CALTAGIRONE C, SPALLETTA G. Anosognosia for hemiplegia after stroke is a multifaceted phenomenon: a systematic review of the literature. Brain 2007; 130: 30753090.

(54) KORTTE K, CHWALISZ K, WEGENER S. Anosognosia and denial: their relationship to coping and depression in acquired brain injury. Rehabilitation psychology 2003; 48(3): 131-136. 